\title{
Use of pegaptanib for recurrent and non-clearing vitreous haemorrhage in proliferative diabetic retinopathy
}

D Hornan, N Edmeades, R Krishnan, J Khan and J Lochhead

\begin{abstract}
Purpose Diabetes is the leading cause of blindness in the United Kingdom among people of working age. Many with proliferative diabetic retinopathy (PDR) go on to develop vitreous haemorrhage (VH). Those with recurrent or non-clearing $\mathrm{VH}$ require vitrectomy to restore vision. Pegaptanib is a vascular endothelial growth factor antagonist that disrupts the proliferative cascade and has been shown to precipitate regression of retinal neovascularisation. We assessed the effect of pre-operative intravitreal (IVT) pegaptanib on the timing, difficulty, and outcome of vitrectomy for recurrent VH in PDR. Methods Fourteen consecutive patients (15 eyes) were given a course of 1-3 IVT pegaptanib injections and vitrectomy was performed when indicated by the recurrence or persistence of $\mathrm{VH}$, or progression of associated tractional retinal detachment (TRD).

Results The range of patient follow-up was from 6 months to 2 years. All had no further VH for at least 4 weeks after IVT pegaptanib. Five eyes remained free from $\mathrm{VH}$ until the end of the study (8-25 months), thus obviating the need for vitrectomy. Two further cases avoided vitrectomy following further IVT pegaptanib. In the majority of patients with VH, IVT pegaptanib created a window for further laser and risk factor optimisation. Surgery was faster and less challenging, compared with conventional vitrectomy for recurrent $\mathrm{VH}$ due to PDR.

Conclusions IVT pegaptanib can be considered in diabetic patients with VH. Approximately one-third may avoid
\end{abstract}

vitrectomy altogether. There are clear intraoperative advantages of using IVT pegaptanib pre-operatively. However, caution should be exercised where there is pre-existing TRD. Eye (2010) 24, 1315-1319; doi:10.1038/eye.2010.14; published online 12 March 2010

Keywords: diabetic retinopathy; pegaptanib; vitreous haemorrhage

\section{Introduction}

Diabetes is a leading cause of blindness in developed countries. Vitreous haemorrhage (VH) due to proliferative retinopathy is a major cause of visual impairment in diabetes. The natural history of $\mathrm{VH}$ is for vision to worsen or show no improvement in more than two-thirds of individuals over a period of 3-10 years. ${ }^{1}$ In approximately half of these individuals, the cause of low vision is non-clearing or recurrent $\mathrm{VH}$.

Recurrent or non-clearing $\mathrm{VH}$ is an established indication for vitrectomy. ${ }^{2}$ The purpose of vitrectomy is to restore visual function, allow further conventional panretinal laser photocoagulation (PRP), and remove the mechanism for tractional retinal detachment (TRD).

Pegaptanib is an anti-vascular endothelial growth factor (VEGF) aptamer that has been shown to inhibit retinal neovascularisation. Pegaptanib is known to be effective in stabilising choroidal neovascularisation due to age-related macular degeneration. ${ }^{3}$ It also causes marked regression of new vessels in proliferative diabetic retinopathy (PDR $)^{4-7}$ and there is growing evidence for its use in diabetic macular oedema. ${ }^{8}$ The safety profile of intravitreal pegaptanib at 3 years is favourable,
Ophthalmology

Department, St Mary's Hospital, Isle of Wight, Newport, Hants, UK

Correspondence:

D Hornan,

Ophthalmology

Department, St Mary's Hospital, Isle of Wight,

Newport, Hants PO20 5TG, UK Tel: + 447734037 619; Fax: + 447092050380

E-mail: dan.hornan@ googlemail.com

Received: 17 August 2009 Accepted in revised form: 14 January 2010 Published online: 12 March 2010 
and its potential applications are widening, particularly with respect to diabetic eye disease. ${ }^{9}$

In diabetic eye disease, there is a theoretical benefit from the selective inhibition of VEGF-A165 by pegaptanib, in contrast to the non-selective inhibition of VEGF-A by antibodies such as ranibizumab and bevacizumab. VEGF-A165 is the pathological isoform in neovascularisation, whereas normal isoforms of VEGF-A have a beneficial role in protection of the retina from ischaemic damage. ${ }^{10}$ In many diabetics, the macular microcirculation is probably already compromised and global VEGF-A inhibition may have a detrimental effect. $^{11-14}$

We present data on the use of intravitreal pegaptanib for recurrent/non-clearing $\mathrm{VH}$ in PDR, in which vitrectomy is conventionally indicated. We assessed the effect of pre-operative intravitreal (IVT) pegaptanib on the timing, difficulty, and outcome of surgery and whether surgery was avoidable in any of these cases.

\section{Materials and methods}

Consecutive patients in whom vitrectomy surgery was indicated by the presence of recurrent or non-clearing diabetic VH were included in the study. Maximum possible PRP was carried out before any intervention. Exclusion criteria were severe TRD within the arcades immediately threatening the fovea, macula off TRD, and extensive preretinal fibrosis and traction.

Each patient was initially administered a single intravitreal pegaptanib injection into the affected eye under topical anaesthesia. Informed consent was obtained and pegaptanib sodium (Macugen, Pfizer, UK) $0.3 \mathrm{mg}$ in $90 \mu \mathrm{l}$ was injected intravitreally with the pre-filled syringe and a 30-gauge needle using aseptic technique. Central retinal artery perfusion was assessed; if not patent then an anterior chamber paracentesis to remove $\sim 0.05 \mathrm{ml}$ of aqueous was performed. Finally, chloramphenicol drops were administered to the treated eye and were continued four times daily for 5 days. Patients were initially followed-up at 2 weeks post-injection and then monthly for the first 6 months.

Following IVT pegaptanib, further PRP was carried out, if possible, as the view cleared. Risk factors for progression of diabetic eye disease were assessed both before and after IVT pegaptanib; appropriate referrals were made to the patient's general practitioner or the hospital diabetic clinic to optimise risk reduction.

Eyes were monitored for further episodes of $\mathrm{VH}$ or non-clearing pre-injection VH. Vitrectomy surgery was carried out if this haemorrhage resulted in significant visual impairment, sufficient to compromise driving, reading, or work, persistently over 2 months or recurrently over 4 months. However, if the posterior hyaloid was still attached or accommodation was intact, a further pegaptanib injection was offered.

The presence of TRD was also recorded at follow-up and ocular coherence tomography (OCT) was used to monitor traction adjacent to the vascular arcades. If TRD threatened the macula, then a vitrectomy was performed. Informed consent was obtained and all vitrectomies were performed by the same surgeon (JL), using a 20-gauge system. Retinal haemostasis with diathermy, endolaser, and segmentation, with or without delamination of scar tissue, was performed as needed. Where retinal breaks were identified, they were treated and retinal tamponade with air or gas used as appropriate.

The cumulative probability of avoiding vitrectomy after IVT pegaptanib was calculated using Kaplan-Meier survival analysis.

\section{Results}

Fourteen consecutive patients (15 eyes) were prospectively recruited to the study (Table 1). There were 5 female eyes and 10 males in the study group, and the age distribution was from 32 to 71 years (mean 52) at the end of the study period. The range of follow-up after the first pegaptanib injection was from 6 months to 2 years.

Regression of new vessels at the disc or elsewhere was observed in all patients in whom the location of the vessels could be visualised, both pre- and post-IVT pegaptanib (Figure 1a and b). Significant clearing of $\mathrm{VH}$ was documented in 12 out of 15 eyes (Figure 1c and d) within the first three visits after IVT pegaptinib. This was accompanied by an improvement in visual acuity in 12 eyes, with 3 eyes maintaining pre-existing acuity

(Table 1). The mean gain in visual acuity for all eyes was $0.8 \log$ MAR units (8 ETDRS lines).

No new VH was noted in all 15 eyes for at least 4 weeks. In 3 out of 15 (20\%) eyes, there was no recurrence of $\mathrm{VH}$, sufficient to cause any visual disability, from the initial pegaptanib injection up until the date of manuscript submission (8-25 months) and these patients had not proceeded to surgery. Four eyes remained free from VH after IVT pegaptanib for a prolonged period (9-22 months), after which vitrectomy was performed for fresh VH. In two eyes (cases 1 and 2) this occurred as a result of posterior vitreous detachment (PVD) causing traction on regressed neovascularisation, rather than re-activation of new vessels.

Two patients (cases 11 and 14) in whom vitrectomy was indicated subsequent to the initial IVT pegaptanib requested further IVT pegaptanib rather than vitrectomy. Both patients had still avoided vitrectomy by the end of the study period ( 6 and 12 months, respectively).

Six eyes had pre-existing TRD before IVT pegaptanib. Progression of TRD was seen in all these eyes. In one eye 
Table 1 Summary of results showing age of subject, type of diabetes mellitus (DM), sex of subject, number of intravitreal (IVT) pegaptanib injections, follow-up $(F / U)$ time in months from 1st IVT pegaptanib, time in months from vitreous haemorrhage to 1st IVT pegaptanib $(\Rightarrow I V T)$, number of PRP laser burns prior to 1st IVT pegaptanib $\left(P R P_{\text {pre }}\right)$, visual acuity pre and post 1st IVT pegaptanib $\left(V A_{\text {pre }}, V A_{p s t}\right)$, VA gain in logMAR units $(V A+)$, number of PRP laser burns subsequent to 1st IVT pegaptanib $\left(P R P_{p s t}\right)$, vitrectomy status $($ Vity, $\mathrm{Y} / \mathrm{N})$, indication for vitrectomy (Indic.), time between most recent IVT pegaptanib and vitrectomy $(\Rightarrow$ Vity, months), and tractional retinal detachment status $(T R D, \mathrm{Y} / \mathrm{N})$

\begin{tabular}{|c|c|c|c|c|c|c|c|c|c|c|c|c|c|c|c|}
\hline Case & Age & $D M$ & Sex & $I V T$ & $F / U$ & $\Rightarrow I V T$ & $P R P_{\text {pre }}$ & $V A_{\text {pre }}$ & $V A_{p s t}$ & $V A+$ & $P R P_{p s t}$ & Vity & Indic. & $\Rightarrow$ Vity & $T R D$ \\
\hline 1 & 32 & 1 & $\mathrm{~F}$ & 3 & 26 & 13 & 8959 & $6 / 36$ & $6 / 12$ & 0.5 & 1342 & $\mathrm{Y}$ & VH & 18 & $\mathrm{~N}$ \\
\hline 2 & 32 & 1 & $\mathrm{~F}$ & 3 & 26 & 10 & 7064 & $6 / 60$ & $6 / 12$ & 0.7 & 1959 & $\mathrm{Y}$ & VH & 22 & $\mathrm{~N}$ \\
\hline 3 & 61 & 2 & $\mathrm{M}$ & 1 & 25 & 1 & 3260 & $6 / 60$ & $6 / 6$ & 1 & 0 & $\mathrm{~N}$ & $\mathrm{~N} / \mathrm{A}$ & $\mathrm{N} / \mathrm{A}$ & $\mathrm{N}$ \\
\hline 4 & 63 & 1 & $\mathrm{M}$ & 1 & 16 & 3 & 831 & CF & $6 / 9$ & 1.8 & 1007 & $\mathrm{Y}$ & VH & 4 & $\mathrm{~N}$ \\
\hline 5 & 69 & 2 & $\mathrm{~F}$ & 1 & 18 & 2 & 3450 & $6 / 60$ & $6 / 60$ & 0 & 0 & $\mathrm{Y}$ & TRD & 1 & Y \\
\hline 6 & 50 & 1 & $\mathrm{~F}$ & 1 & 16 & 1 & 3271 & CF & $6 / 9$ & 1.8 & 0 & $\mathrm{Y}$ & TRD & 1 & Y \\
\hline 7 & 34 & 1 & $\mathrm{~F}$ & 1 & 22 & 10 & 7418 & $6 / 60$ & $6 / 12$ & 0.7 & 0 & Y & TRD & 1 & Y \\
\hline 8 & 57 & 2 & $\mathrm{M}$ & 1 & 12 & 5 & 3766 & $6 / 60$ & $6 / 12$ & 0.7 & 2369 & $\mathrm{Y}$ & VH & 9 & Y \\
\hline 9 & 69 & 2 & $\mathrm{M}$ & 1 & 12 & 72 & 6442 & $6 / 6$ & $6 / 6$ & 0 & 0 & $\mathrm{~N}$ & N/A & N/A & $\mathrm{N}$ \\
\hline 10 & 35 & 1 & $\mathrm{M}$ & 1 & 11 & 2 & 5703 & $6 / 9$ & $6 / 4$ & 0.4 & 489 & Y & VH & 11 & $\mathrm{~N}$ \\
\hline 11 & 67 & 2 & $\mathrm{M}$ & 2 & 11 & 1 & 2699 & CF & $6 / 12$ & 1.7 & 3429 & $\mathrm{~N}$ & $\mathrm{~N} / \mathrm{A}$ & $\mathrm{N} / \mathrm{A}$ & $\mathrm{N}$ \\
\hline 12 & 35 & 1 & $\mathrm{M}$ & 1 & 11 & 5 & 7920 & $6 / 240$ & $6 / 36$ & 0.8 & 0 & $\mathrm{Y}$ & TRD & 1 & Y \\
\hline 13 & 62 & 2 & $\mathrm{M}$ & 1 & 8 & 1 & 5687 & $6 / 9$ & $6 / 9$ & 0 & 1002 & $\mathrm{~N}$ & $\mathrm{~N} / \mathrm{A}$ & $\mathrm{N} / \mathrm{A}$ & Y \\
\hline 14 & 71 & 2 & $\mathrm{M}$ & 2 & 6 & 51 & 987 & CF & $6 / 60$ & 1 & 0 & $\mathrm{~N}$ & $\mathrm{~N} / \mathrm{A}$ & $\mathrm{N} / \mathrm{A}$ & $\mathrm{N}$ \\
\hline 15 & 39 & 1 & $\mathrm{M}$ & 1 & 6 & 1 & 9625 & $6 / 18$ & $6 / 12$ & 0.2 & 0 & $\mathrm{Y}$ & TRD & 1 & Y \\
\hline
\end{tabular}
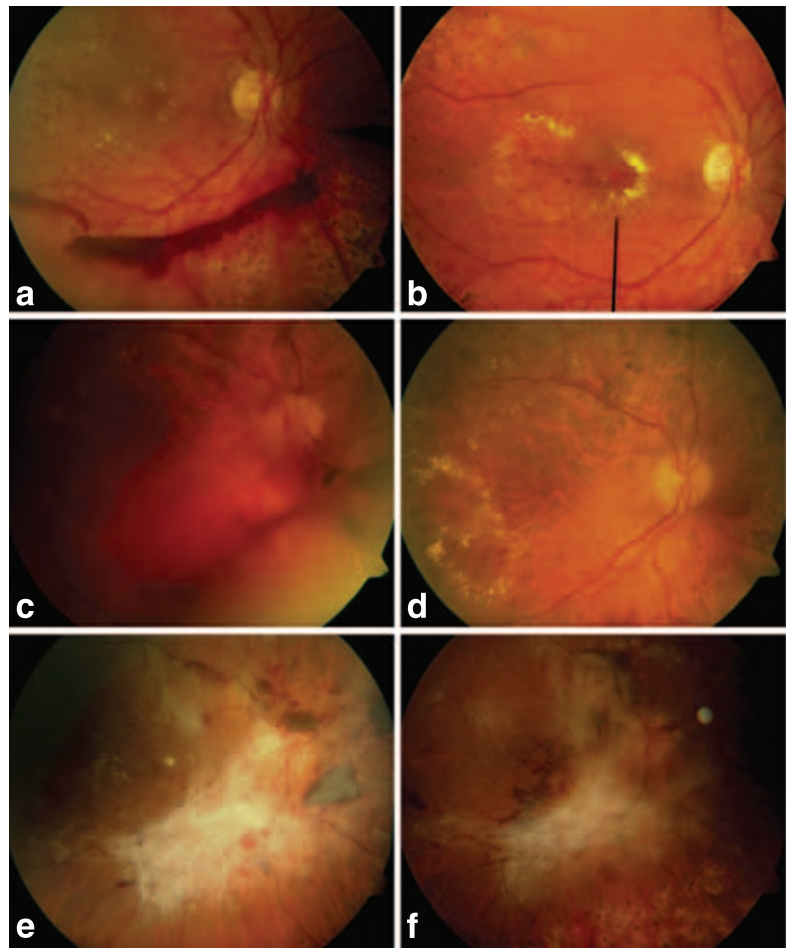

Figure 1 Colour fundus photographs of illustrative cases before and after intravitreal pegaptanib. (a and b) (case 2) Regression of extensive neovascularisation. Similar regression was observed in all eyes in which a good view was obtainable both before and after IVT pegaptanib. (c and d) (case 13) Clearing of vitreous haemorrhage $(\mathrm{VH})$. Cessation of $\mathrm{VH}$ was observed in 12 out of 15 eyes after IVT pegaptanib for at least 4 weeks. (e and f) (case 6) Contraction of scar tissue associated with increased macular detachment. Progression was seen in all eyes with pre-existing TRD, although it remained outside the arcades if it had not reached them before IVT pegaptanib. (case 12), TRD was observed for the first time after clearing of VH subsequent to IVT pegaptanib. TRD present outside the macula before IVT pegaptanib (cases 8 and 13) remained so after treatment. In eyes with preexisting TRD within the arcades (but not immediately threatening the macula), contraction of scar tissue was associated with increased macular detachment both clinically (Figure 1e and f) and on OCT. This was observed within 1 month of IVT pegaptanib and all cases urgently proceeded to vitrectomy.

Subsequent to IVT pegaptanib, 10 out of 15 (67\%) eyes required vitrectomy due to recurrent/non-clearing $\mathrm{VH}(5$ eyes) or TRD threatening the macula (5 eyes). It was the opinion of the same experienced surgeon that vitrectomy was technically easier, faster, and less haemostasis was required compared with similar procedures without IVT pegaptanib pre-treatment.

The Kaplan-Meier survival curve (Figure 2), plotted from the cumulative probability of avoiding vitrectomy, shows a marked initial reduction in probability followed by a more gradual decline. The drop in survival probability within 6 weeks of injection represents vitrectomy for progression of TRD, whereas the gradual decline over the following 2 years corresponds to recurrent or non-clearing $\mathrm{VH}$.

\section{Discussion}

All patients showed regression of new vessels where they could be monitored both before and after IVT pegaptanib. This is consistent with previous studies where pegaptanib has been used in diabetic eyes, both as 


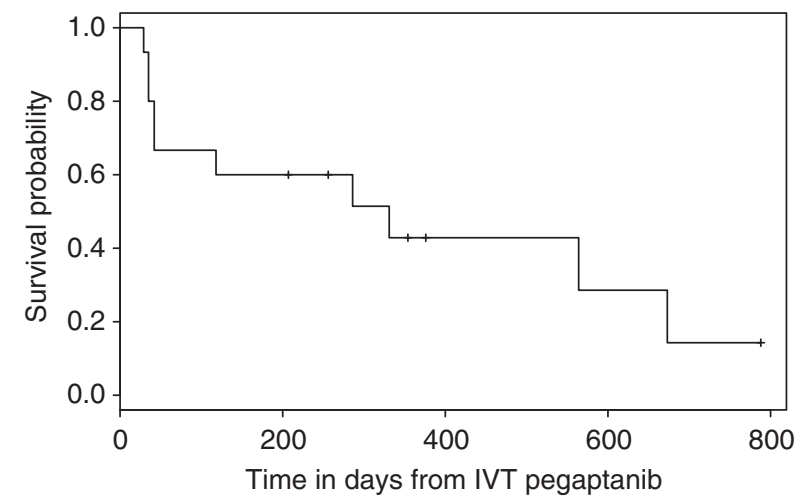

Figure 2 Survival curve showing probability of avoiding vitrectomy or further intravitreal (IVT) pegaptanib after a single IVT pegaptanib treatment for recurrent vitreous haemorrhage.

a retrospective finding in eyes treated for diabetic macular oedema and when pegaptanib was given specifically for PDR. ${ }^{4-7}$ IVT pegaptanib has also been successfully used to treat bilateral $\mathrm{VH}$ in idiopathic retinal vasculitis (Eales Disease), with clearance of $\mathrm{VH}$ and prevention of further $\mathrm{VH}$ for at least 9 months postinjection. ${ }^{15}$

The anti-VEGF antibodies ranibizumab and bevacizumab have been used as intravitreal adjuncts before vitrectomy for recurrent/non-clearing diabetic VH. However, in the majority of cases, surgery was carried out within 2 weeks of intravitreal anti-VEGF. ${ }^{16-18}$ An early report of two cases with VH due to PDR showed rapid resolution following intravitreal bevacizumab. ${ }^{19}$ More recently, two studies of IVT bevacizumab for PDR reported resolution of $\mathrm{VH}$ in five eyes. This was for at least 3 months post-treatment in two eyes and at least 6 months post-treatment in three eyes. ${ }^{20,21}$ One recent study of 20 -week follow-up after intravitreal bevacizumab that specifically targeted active progressive PDR (including $\mathrm{VH}$ ) showed significant improvement of best-corrected visual acuity and density of $\mathrm{VH}$ in the study group as a whole. ${ }^{22}$

We have demonstrated stabilisation of diabetic $\mathrm{VH}$ in those cases where surgery is indicated. A significant initial clearance of $\mathrm{VH}$ occurred in all patients, accompanied by a marked improvement in visual acuity in the vast majority of cases. This period of stability created a window for further PRP, where possible, in addition to optimisation of diabetic and blood pressure control. In the younger diabetic patients with an attached posterior hyaloid, this delay allowed time for PVD to occur, making surgery technically easier and safer.

A third of our patients avoided surgery altogether. Once again, this is particularly beneficial in younger patients with residual lens accommodation, given the high probability of cataract formation subsequent to vitrectomy. A significant proportion of patients did ultimately require vitrectomy, even after a prolonged period of remission. However, in some cases this resulted from PVD or traction (either on previously inactive new vessels or the macula), rather than disease reactivation.

The most severe cases of TRD were excluded from the study, as it was felt that administration of IVT pegaptanib may result in a retinal tear, given the fibrotic contraction which can occur. ${ }^{23}$ Indeed, we observed progression of TRD after IVT pegaptanib in all cases with pre-existing TRD, and this was the indication of all vitrectomies within 6 weeks of injection. There are several reports of similar or more severe progression after the use of IVT anti-VEGF antibodies. ${ }^{21,22,24}$ IVT anti-VEGF must be used with extreme caution in patients with TRD close to the vascular arcades. Further studies are needed to compare progression of TRD after pegaptanib with that seen after non-specific anti-VEGF-A therapies. ${ }^{10}$

This study has limitations of relatively small numbers, lack of a control group, and no randomisation. However, consecutive patients meeting specific criteria were included in the study and followed for many months, thus reducing selection bias. This study highlights some of the advantages and pitfalls to the use of IVT pegaptanib in severe and non-clearing diabetic vitreous haemorrhage.

\section{Summary}

What was known before

- Pegaptanib causes regression of neovascularisation.

- Vitreous haemorrhage is a disabling complication of proliferative diabetic retinopathy.

What this study adds

- Pegaptanib may be useful in treating vitreous haemorrhage in PDR.

- At the very least, it provides a window of opportunity for conservative treatment.

\section{Conflict of interest}

The authors declare no conflict of interest.

\section{References}

1 Ziemianski MC, McMeel JW, Franks EP. Natural history of vitreous hemorrhage in diabetic retinopathy. Ophthalmology 1980; 87(4): 306-312.

2 Yang $\mathrm{CM}$. Surgical treatment for diabetic retinopathy: 5-Year experience. J Formos Med Assoc 1998; 97(7): 477-484.

3 Gonzales CR, VEGF Inhibition Study in Ocular Neovascularization (VISION) Clinical Trial Group. Enhanced efficacy associated with early treatment of neovascular age-related macular degeneration with 
pegaptanib sodium: An exploratory analysis. Retina 2005; 25(7): 815-827.

4 Adamis AP, Altaweel M, Bressler NM, Cunningham ET, Davis MD, Goldbaum $\mathrm{M}$ et al. Changes in retinal neovascularization after pegaptanib (macugen) therapy in diabetic individuals. Ophthalmology 2006; 113(1): 23-28.

5 Krzystolik MG, Filippopoulos T, Ducharme JF, Loewenstein JI. Pegaptanib as an adjunctive treatment for complicated neovascular diabetic retinopathy. Arch Ophthalmol 2006; 124(6): 920-921.

6 Bansal AG, Narayanan R, Majii AB, Thomas R. Neovascular changes after pegaptanib in diabetics. Ophthalmology 2007; 114(3): 615-616.

7 Mendrinos E, Donati G, Pournaras CJ. Rapid and persistent regression of severe new vessels on the disc in proliferative diabetic retinopathy after a single intravitreal injection of pegaptanib. Acta Ophthalmol 2009; 87(6): 683-684.

8 Cunningham ET, Adamis AP, Altaweel M, Aiello LP, Bressler NM, D'Amico DJ et al. A phase II randomized double-masked trial of pegaptanib, an anti-vascular endothelial growth factor aptamer, for diabetic macular edema. Ophthalmology 2005; 112(10): 1747-1757.

9 Singerman LJ, Masonson H, Patel M, Adamis AP, Buggage $\mathrm{R}$, Cunningham $\mathrm{E}$ et al. Pegaptanib sodium for neovascular age-related macular degeneration: Third-Year safety results of the VEGF inhibition study in ocular neovascularisation (VISION) trial. Br J Ophthalmol 2008; 92(12): 1606-1611.

10 Nishijima K, Ng YS, Zhong L, Bradley J, Schubert W, Jo N et al. Vascular endothelial growth factor-a is a survival factor for retinal neurons and a critical neuroprotectant during the adaptive response to ischemic injury. Am J Pathol 2007; 171(1): 53-67.

11 Chen E, Hsu J, Park CH. Acute visual acuity loss following intravitreal bevacizumab for diabetic macular edema. Ophthalmic Surg Lasers Imaging 2009; 40(1): 68-70.

12 Neubauer AS, Kook D, Haritoglou C, Priglinger SG, Kampik A, Ulbig MW et al. Bevacizumab and retinal ischemia. Ophthalmology 2007; 114(11): 2096.

13 Kim KS, Chang HR, Song S. Ischaemic change after intravitreal bevacizumab (avastin) injection for macular oedema secondary to non-ischaemic central retinal vein occlusion. Acta Ophthalmol 2008; 86(8): 925-927.
14 Chung EJ, Roh MI, Kwon OW, Koh HJ. Effects of macular ischemia on the outcome of intravitreal bevacizumab therapy for diabetic macular edema. Retina 2008; 28(7): 957-963.

15 Mitry D, Schmoll C, Hegde V, Borooah S, Singh J, Bennett H. Use of pegaptanib in the treatment of vitreous haemorrhage in idiopathic retinal vasculitis. Eye 2008; 22(11): 1449-1450.

16 Yang CM, Yeh PT, Yang CH, Chen MS. Bevacizumab pretreatment and long-acting gas infusion on vitreous clearup after diabetic vitrectomy. Am J Ophthalmol 2008; 146(2): 211-217.

17 Yeoh J, Williams C, Allen P, Buttery R, Chiu D, Clark B et al. Avastin as an adjunct to vitrectomy in the management of severe proliferative diabetic retinopathy: a prospective case series. Clin Experiment Ophthalmol 2008; 36(5): 449-454.

18 Ishikawa K, Honda S, Tsukahara Y, Negi A. Preferable use of intravitreal bevacizumab as a pretreatment of vitrectomy for severe proliferative diabetic retinopathy. Eye 2009; 23(1): 108-111.

19 Spaide RF, Fisher YL. Intravitreal bevacizumab (avastin) treatment of proliferative diabetic retinopathy complicated by vitreous hemorrhage. Retina 2006; 26(3): 275-278.

20 Minnella AM, Savastano CM, Ziccardi L, Scupola A, Falsini B, Balestrazzi E. Intravitreal bevacizumab (avastin) in proliferative diabetic retinopathy. Acta Ophthalmol 2008; 86(6): 683-687.

21 Arevalo JF, Wu L, Sanchez JG, Maia M, Saravia MJ, Fernandez $\mathrm{CF}$ et al. Intravitreal bevacizumab (avastin) for proliferative diabetic retinopathy: 6-months follow-up. Eye 2009; 23(1): 117-123.

22 Moradian S, Ahmadieh H, Malihi M, Soheilian M, Dehghan $\mathrm{MH}$, Azarmina M. Intravitreal bevacizumab in active progressive proliferative diabetic retinopathy. Graefes Arch Clin Exp Ophthalmol 2008; 246(12): 1699-1705.

23 Krishnan R, Goverdhan S, Lochhead J. Intravitreal pegaptanib in severe proliferative diabetic retinopathy leading to the progression of tractional retinal detachment. Eye 2009; 23(5): 1238-1239.

24 Tranos P, Gemenetzi M, Papandroudis A, Chrisafis C, Papadakos D. Progression of diabetic tractional retinal detachment following single injection of intravitreal avastin. Eye 2008; 22(6): 862 